\title{
Methods and Techniques for Brain Image Segmentation
}

\author{
Greeshma K V \\ Asst. Professor \\ Department of Computer Science \\ Carmel College Mala \\ Thrissur, India
}

\begin{abstract}
Image Segmentation is a method of dividing a group of data into different segments which has uniform characteristics. It is a computational process done by automatically and broadly used in medical imaging for the diagnosis and treatment of various diseases in human body. Brain is the supreme part of central nervous system which controls and coordinating all the activities of the body. Analyzing the complex structure of brain and finding the abnormalities is very challenging and time consuming task for medical practitioners. So brain image segmentation is very important but most challenging problem among medical images. This paper depicts the different challenges and applications of brain image segmentation which can be extended in many directions of brain images. In this paper we review the different modalities of medical images like MRI, CT, and SPECT etc. We highlight the various applications of these modalities which are used in image registration, detection and retrieval for the purpose of proper diagnosis of brain disorders.
\end{abstract}

Keywords - Image Segmentation, Brain Images, MRI Brain Images, SPECT Brain Images.

\section{INTRODUCTION}

In medical imaging technologies, images obtained from the different modalities like MRI, and X-ray representing the soft tissue, organs and visualization of human body. One of the important tasks in medical imaging is brain tumor segmentation. Early diagnosis of brain tumor plays an important role in the survival rate of patients and treatment possibilities. Brain tissue segmentation is used for the detection and diagnosis of normal and pathological tissues abnormalities or tumors. Manual segmentation of brain tumors is a very difficult and time consuming task. There is a need for automatic brain tumor image segmentation [6].

Tumor is an uncontrolled, unnatural cell growth and division in the brain tissue. It is a type of cancer and not very common. One type of brain tumors are gliomas which originate from glial cells. Currently brain tumor segmentation research focuses on these types of brain tumors. Early diagnosis of gliomas plays an important role in improving treatment possibilities. Medical Imaging techniques such as Computed Tomography (CT), Single-Photon Emission Computed Tomography (SPECT), Positron Emission Tomography (PET), Magnetic Resonance Spectroscopy (MRS) and Magnetic Resonance Imaging (MRI) are all used to provide valuable information about shape, size, location and metabolism of brain tumors assisting in diagnosis. While these modalities are used in combination to provide the highest detailed information about the brain tumors, due to its good soft tissue contrast and widely availability MRI is considered as the standard technique [6].

An accurate segmentation of brain images is a very difficult task. On the other hand, in most cases an accurate and precise segmentation is crucial for a correct diagnosis by clinical tools. Moreover, manual segmentation of brain MRI images is a time-consuming and labor-intensive procedure; therefore, automatic image segmentation is widely used for this purpose [6]. We briefly review the different methods, which are proposed in recent year's brain image segmentation. Finally, in conclusions, we evaluate the current state-of-the-art and provide future directions for development.

\section{BRAIN IMAGES}

The rapid development of different kinds of highly equipped medical instruments and more use of such images have made it difficult for the medical experts to interpret and infer correct diagnosis. Complicated image features, eye fatigue are the factors that may cause an expert to miss an abnormality in an image. Hence, there is a great need for robust methods that process with the interpretation of huge amounts of data with greater accuracy. To alleviate these difficulties in clinical diagnosis, segmentation of medical images provides the potentiality for increasing the diagnostic accuracy.

Magnetic Resonance Imaging (MRI) is a well-established non-invasive diagnostic medical imaging technique based on the nuclear magnetic resonance phenomenon. Although qualitative image analysis is often sufficient for diagnosis of diseases, quantitative analysis is necessary for many applications, for which segmentation is a primary step. Segmentation of MR Images is a challenging problem due to its complexity as well as to the absence of models of the anatomy that fully capture the possible deformations in each structure. Conventional MRI relies on a difference in a weighted average of spectral and temporal information from tissue to tissue to make a diagnosis. The intensity of the MR image of human tissue is homogeneous and the structure of each tissue is connected, but is difficult to separate the adjacent tissue due to the small intensity changes and smoothed boundaries between tissues. Further, the lacks of clearly defined edges includes intra and inter observer variability, which deteriorates the significance of the analysis. Brain tissue is a complex structure. The diagnosis of many 
brain disorders involves accurate tissue segmentation of brain MR images.

\section{BRAIN SCANNING IMAGES}

Brain imaging has greatly advanced in the last 20 years, due to better understanding of the electromagnetic spectrum and radiofrequency waves, in relation to protons in individual molecules within the cells of the brain.

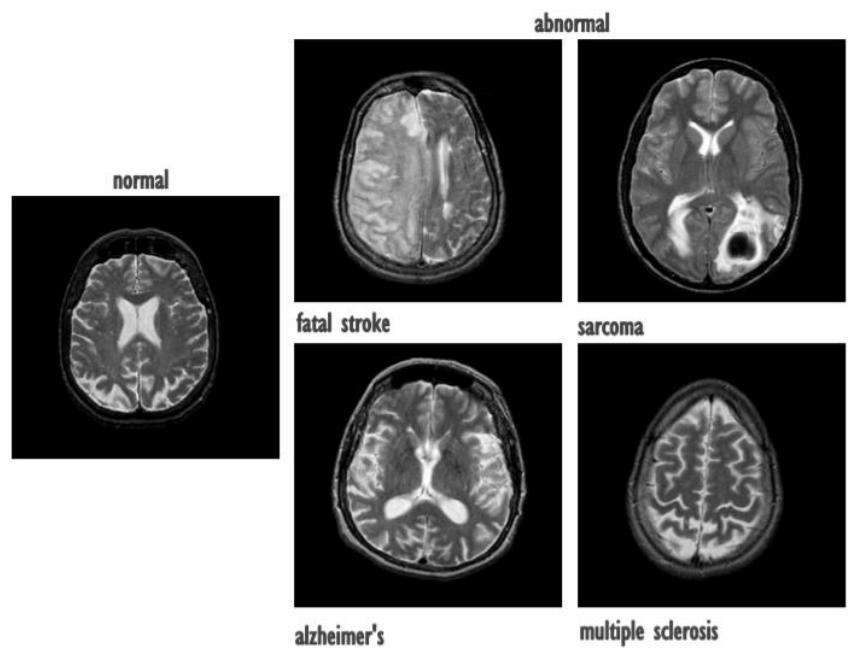

Fig 1: Samples of T2- weighted control and abnormal brain MR images [5]

New technologies allow non-invasive spatial mapping, (morphology), and observations of processes within the brain during set tasks. By sequencing scanned sections of the brain, activity between neurons in different parts of the brain can be observed and monitored. More recent technologies using a higher frequency resolution can identify the distribution of individual metabolites (large complex molecules), and pharmaceutical drugs. There are a range of scanning techniques, their purpose and limitations are described below:

\section{A. Positron emission tomography (PET)}

Positron emission tomography scanning produces a threedimensional image of functional processes in the brain, (not just the structure). PET is a nuclear medicine imaging technique which requires the patient to receive a small injection of radio-active material (a sugar tracer; fluoro deoxy glucose), into the bloodstream. The radio-active material causes the production of gamma-rays, these are a form of electromagnetic radiation like X-rays, but of higher energy. The areas of the brain that command the greater volumes of blood produce the most gamma-rays, and it is these areas that are computed and displayed by the PET scan. This system not only identifies the activated area of the brain, but also measures the degree of activity.

\section{B. Magnetic resonance imaging (MRI)}

An MRI scanner uses a strong magnetic field and radio waves to create pictures of the tissues and other structures inside the brain, on a computer. The magnetic field aligns the protons (positively charged particles) in hydrogen atoms, like tiny magnets. Short bursts of radio waves are then sent to knock the protons out of position, and as they realign, (relaxation time), they emit radio signals which are detected by a receiving device in the scanner. The signals emitted from different tissues vary, and can, therefore, be distinguished in the computer picture.

MR imaging tool has different advantages including imaging in different directions and also it is relatively safe as compared to the other imaging modalities such as Computer Tomography (CT) and X-ray. MRI is used to capture images in different modalities such as T1-weighted, T2-weighted, and Proton Density (PD)-weighted. This capability help to better diagnose the different diseases such as cancers and Multiple Sclerosis (MS).

An MRI scanner can create clear detailed pictures of the structure of the brain and detect any abnormalities or tumors. The relaxation times, $\mathrm{T} 1, \mathrm{~T} 2$, and $\mathrm{T} 2 *$ are measured after the scanner's pulse sequence, and can be chosen to look at specific tissue within the brain. It may be seen that by selecting different relaxation times and manipulating radio frequencies, specific brain tissue can be highlighted for examination by the physician.

The process of having a scan is painless and safe, there is no exposure to radiation, but occasionally, a patient may have a reaction to the tracer dye. Pregnant mothers are not recommended to undertake the procedure unless there is no alternative, since it is not known whether the effects of a strong magnetic field may affect the developing baby. The patient may resume normal activities immediately after the scan, and the radiologist studies the pictures and sends a report to the doctor.

\section{Computerised tomography (CT)}

Computerized tomography scans use X-rays to show the structure of the brain, with details such as blood perfusion, (plates a and b), the resultant images are two dimensional and of comparatively low resolution, however, the quality has been much improved since 1998. With improved technology, the single section has now become a multisection and the speed has increased eight times, giving well-defined 3-D pictures. A CT scan may reveal underdeveloped parts of the brain or sites of injury from impact, tumors, lesions or infection.

Before a CT scan, the patient may drink but is asked not to eat for four hours beforehand, and not to take strenuous exercise. A CT brain scan will take about 30 minutes and the patient must lie still for the duration. The radiologist needs to know if the patient is diabetic, pregnant or on medication. The procedure is painless, but does involve exposure to radiation at a very low level.

\section{Single photon emission computed tomography (SPECT)}

The single photon emission computed tomography records the signals from gamma rays, (singly, rather than when the emissions are opposite at $180^{\circ}$ ), using two or more synchronized gamma cameras, and the multiple 2-D images are computed, tomographically reconstructed, to 3-D. A section may be examined from several angles, but is slightly less clear than a PET image.

A SPECT scanner is less expensive than a PET scanner and uses longer-lived, more easily obtained radioisotopes. Tracing blood flow within the brain identifies where metabolic activity is occurring, enabling assessment of brain functions. The patient will not have to fast before the 
procedure, but will have to remain absolutely still for 15 to 20 minutes in a scanner, similar to the MRI. The procedure is painless and the patient may resume normal activities immediately afterwards.

\section{METHODS FOR BRAIN TUMOR IMAGE SEGMENTATION}

Brain tumor segmentation methods can be classified as manual methods, unsupervised methods, semi-supervised and supervised methods.

\section{A. Manual Segmentation Methods}

In manual segmentation methods, the radiologist analyzing the information presented by various scan images and manually diagnosing the tumor and drawing the tumor regions carefully by going through different slices of image slice by slice. This is a time consuming task and it needs a lot of experience and training. Manual segmentation is a radiologist dependent method.

\section{B. Unsupervised Methods}

Unsupervised methods do not use prior labels for image segmentation process. Most commonly used two categories in this method are Fuzzy C-Means (FCM) and Finite Mixture Model (FMM). Other supervised methods like SelfOrganizing Maps (SOM) and Neural Networks methods are also used for segmentation of brain images. Clustering methods are the most common methods used for image segmentation.

\section{Semi-supervised and Supervised Methods}

In these types of methods they use prior class labels for image segmentation. Atlas based methods and machine learning based methods are the two main categories in this method. Neural Network methods like MLP (Multi-Layer Perceptron) and SVM (Support Vector Machine) are the two common machine learning approaches used in this area.

\section{IMAGE SEGMENTATION}

The goal of image segmentation is to divide an image into a set of semantically meaningful, homogeneous, and nonoverlapping regions of similar attributes such as intensity, depth, color, or texture. The segmentation result is either an image of labels identifying each homogeneous region or a set of contours which describe the region boundaries.

Fundamental components of structural brain MRI analysis include the classification of MRI data into specific tissue types and the identification and description of specific anatomical structures. Classification means to assign to each element in the image a tissue class, where the classes are defined in advance. The problems of segmentation and classification are interlinked because segmentation implies a classification, while a classifier implicitly segments an image. In the case of brain MRI, image elements are typically classified into three main tissue types: white matter (WM), gray matter (GM), and cerebrospinal fluid (CSF). The segmentation results are further used in different applications such as for analyzing anatomical structures, for studying pathological regions, for surgical planning, and for visualization.
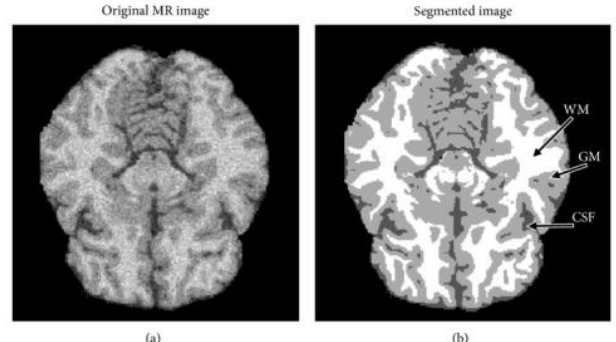

Fig 2: Samples of original and segmented image
Image segmentation can be performed on 2D images, sequences of $2 \mathrm{D}$ images, or $3 \mathrm{D}$ volumetric imagery. Most of the image segmentation research has focused on $2 \mathrm{D}$ images. If the data is defined in 3D space (e.g., obtained from a series of MRI images), then typically each image "slice" is segmented individually in a "slice-by-slice" manner. This type of segmenting 3D image volumes often requires a postprocessing step to connect segmented $2 \mathrm{D}$ slices into a $3 \mathrm{D}$ volume or a continuous surface. Furthermore, the resulting segmentation can contain inconsistencies and nonsmooth surface due to omitting important anatomical information in $3 \mathrm{D}$ space. Therefore, the development of $3 \mathrm{D}$ segmentation algorithms is desired for more accurate segmentation of volumetric imagery.

\section{CONCLUSION}

Image segmentation is an important step in many medical applications and automatic segmentation of the brain tumors for cancer diagnosis is a challenging task. The availability of public datasets like BRATS benchmark provides a medium for researchers to develop and evaluate their models with the existing techniques. This paper has provided a brief introduction to the different modalities that are commonly used and various methods of segmentation of the human brain. Consequently, medical image segmentation and especially brain segmentation issue is an open problem which needs to be more accurate and precise than the other non-medical image segmentation applications.

\section{ACKNOWLEDGEMENT}

We would like to thank the Carmel College Mala from their interest and support to this seminar and, personally, to the person Ms. Smitty V Isidhore, Asst. Professor, Carmel College Mala.

\section{REFERENCES}

[1] Ahmadvand, A., \& Daliri, M. (2014). Brain MR image segmentation methods and applications. OMICS J Radiol, 3(4), e130.

[2] Anthea Wright, Brain scanning techniques (CT, MRI, fMRI, PET, SPECT, DTI, DOT), https://psicoterapiabilbao.es/wpcontent/uploads/2015/12/Brain_scanning_techniques.pdf

[3] Bakas, S., Reyes, M., Jakab, A., Bauer, S., Rempfler, M., Crimi, A., .. \& Prastawa, M. (2018). Identifying the best machine learning algorithms for brain tumor segmentation, progression assessment, and overall survival prediction in the BRATS challenge. arXiv preprint arXiv: 1811.02629 .

[4] Despotović, I., Goossens, B., \& Philips, W. (2015). MRI segmentation of the human brain: challenges, methods, and applications. Computational and mathematical methods in medicine, 2015.

[5] Gudigar, A., Raghavendra, U., San, T. R., Ciaccio, E. J., \& Acharya, U. R. (2019). Application of multiresolution analysis for automated 
detection of brain abnormality using MR images: A comparative study. Future Generation Computer Systems, 90, 359-367.

[6] Işın, A., Direkoğlu, C., \& Şah, M. (2016). Review of MRI-based brain tumor image segmentation using deep learning methods. Procedia Computer Science, 102, 317-324.

[7] Senthilkumaran, N., \& Rajesh, R. (2011). Brain image segmentation. International journal of wisdom based computing, 1(3), 14-18.

[8] Yanase, J., \& Triantaphyllou, E. (2019). The seven key challenges for the future of computer-aided diagnosis in medicine. International journal of medical informatics. 